plain and distinct answer; an answer of facts and of dates, not of conjecture and declamation. What then, I would ask, has Sir William Burnett done for the navy, or for the surgeons of the navy, since he was placed at their head ?--C.

FITTEST PRESENT TO SIR WILLIAM BURNETT.

To the Edilor of The Laxcet.

SIR,-I dislike contributing money for purposes similar to that to which the attention of my brother officers is now solicited by the Portsmouth Committee, because I have on every similaroccasion heard remarks such as the following:- "Well, such and such a one may be very deserving, but I cannot spare the money without injury to my family; and yet if I do not, I suppose I shall be looked upon as a black sheep." Another says, "It is well for a set of men who have already good situations, and who can afford to subscribe, to endeavour to carry themselves into still better, at the ex. pense of us who have received no benefits but what we have deserved, or who, as some say, have not got our deserts."

I do not argue at all, Mr. Editor, for the candour, liberality, or justice of such remarks, but I have repeatedly heard them, and I do say, that subscriptions set on foot for such purposes, always engender much bad feeling, and, in a moral point of view, benefit neither the receiver nor the donor, and had therefore better be let alone.

It may be said, But why not reward merit? and I say so too, but then let the reward be offered at a time when a reflection cannot be made; when no one can be thought to contribute grudgingly, and when no suspicion of impure motives can be attached to the donors- to even the Committee. The time $I$ allude to, is when the person retires from office. At that period, Sir, should I live to see it, I, for one, shall be happy to contribute towards an " esteem present" to Sir William Burnett, provided he continues to perform his duty in the efficient manner I conceive he has performed it hitherto ; though for various reasons I am far from thinking that every person in office who does his duty, should be rewarded so liberally as the Committee propose.

Having thus, Sir, expressed my thoughts on this subject, I again repeat (and I repeat it as a man indebted to no person living for any-thing more than I have justly merited), that I believe Sir William Burnett has done his duty towards the corps, and this expression of my opinion is the only " esteem present" which, from conscientious motives I can think of contributing.

I am, Sir, your obedient servant, A. Courtney, Surg. R,N. Broadstairs, Oct. 3d, 1831.

\section{TREATIEN'T OF EPILEPSY}

WITH THE DIGITALIS AND POLYPODIUM.

\section{By A. Courtney, Esq., Surgeon, R.N., Broadstairs, Kent.}

Having read in The Lancet, of the 27 th of August last, an account by Dr. Sharkey of sereral cases of epilepsy which were treated, successfully, by the digitalis and polypodium, I beg to state, through the medium of the same invaluable publication, for the further encouragement of the pro. fession to extend the trial of those reme. dies, that I had a friend cured of that complaint by them in the summer of 1802 , who has never since had a return of it, and who is now in good bealth.

What the co-existent condition of the internal viscera was, in this case, I am unable to say, and am also incapable of forming an opinion of the cause which gave origin to the disease, having at the time not commenced my professional studies; suffice it to state, neither himself nor relations could assigg any particular cause, and he had previously enjoyed uninterrupted good health. The patient was of a sanguine temperament, was first attacked in his sixteenth year, and had, from that age until. he was twenty-five, frequent, though not very regularly-recurring, fits; sometimes once a month, occasionally at shorter intervals, but never seldomer than once in ten weeks. The following was the formula used :-

Recent leaves of digitalis, four ounces, infused for twenty-four hours in a pint of boiling water. When strained this was divided into three doses, one of which was ordered to be taken every third day with fifteen grains of the dried leaves of poly. pody in powder.

But such was the effect of the first dose, that his relations would not permit him to take a second, for, a few minutes after he had taken it, a vomiting commenced, which, in spite of every-thing that could be done to allay it, continued almost incessantly for five days, accompanied with such prostration of strength, that it was thought at times doubtful whether he was dead or alive. The remedy was recommended by a countryman who was said to be very successful in the treatment of such cases.

Though the exciting causes of epilepsy are so numerous that it would be in vain to expect invariable success from any one description of medicines whatever, yet as the case just related, and the several other cases mentioned by Dr. Sharkey, prove fully that the remedy in question has cured many, after every othes means had failed, 
practitioners should, I think, not be de- the circumstance noticed in any writers on terred from giving it a trial in a disease in the subject. I am, Sir, which, as the friends of Dr. Sharkey's patients expressed themselves, death, should it follow, would be preferable to its continuance; and when we further consider, that though some of that gentleman's patients seemed alarmingly affected by the medicine, yet that none of them died, we have, I think, good reason not to dread, greatly, its effects.

In the case just related, every remedy that the most experienced practitioners could suggest was tried previously, and it will be observed that the dose exhibited was considerably (and probably unnecessarily) stronger than any given by Dr. Sharkey.

Broadstairs, Oct. 1831.

PRODUCTION OF

\section{PUSTULES ON THE TONGUE}

BY THE EXHIBITION OF LARGE DOSES OF TARTRATE OF ANTIMONY.

\section{To the Editor of The Lancet.}

Sir,- $\mathrm{I}$ have, in inflammatory affections of the lungs, been in the practice of prescribing, upon the Italian and French plan of treatment, the tartrate of antimony, in very considerable doses; viz. from ten to thirty grains in the day, in solution, generally with good effect. My opportunities of observation are numerous, as inflammatory diseases of the chest are frequent in this city from the excessive atmospheric moisture that prevails at almost all seasons, and as the duty of attending all acute diseases, with very few exceptions, requiring gratuitous aid, devolves on the dispensary to which $I$ have been attached for the last five years, my practice has been extensive. Aiter some days use of the antimonial, I have occasionally met with pustular eruptions of the tongue and lining membrane of the mouth, of the character, to all appearance, of the pustules produced on the skin by the application of the ointment of the tart. ant., and that without any previous excoriation. In the last case in which I observed it, the tongue only was affected; the medicine was discontinued and the pustules healed in two days; but the inflammatory action still going on, the use of the solution was resumed, and again pustules were produced.

My object in this communication is, to elicit the observations of others more practised and more competent. I have not seen
Your obedient servant,

War. Flemyng Porter, M.D. One of the Physicians to the Cork General Dispensary.

Cork, September 24, 1331.

EXTERNAI, APPLICATION OF THE

\section{CHLORIDE OF SULPHUR IN CUTANEOUS AFF ECTIONS.}

\section{To the Editor of The Lancet.}

Sir,-I shall feèl obliged by your insert. ing the following outlines of cutaneous aftections cured by the external application of the chloride of sulphur, a remedy scarcely noticed as a therapeutic agent. The beneficial effects resulting from its use for these fourteen months, induce me to intrude upon your valuable pages as the best means of giving it publicity.

I am, Sir, your obedient servant, J. Pelfam Buckiand. 20, Great Trinity-lane, Oct. 1831.

CASE.

A young gentleman, who suffered soverely from a periodical psoriasis for many years, consulted me, September 29, 1830, for the above disease, which had attacked him six weeks previously; during that time he employed several means to eradicate it, without effect; the leg was entirely covered with scales and deep fissures pouring out an acrid discharge; the thigh and arm were in a slight degree affected. I ordered him alterative doses of the pilul. bydrarg. with gentle saline aperients, and to rub the parts affected with an ointment composed of $z^{i}$ of the chloride of sulphur, to $z^{i}$ af simple cerate; he continued this treatment for a week, when he was completely cured, and remained so until the beginning of September in the present year, when he used the ointment for a few days, and is again quite recovered.

In December, 1830, a medical friend sent me a young woman, affected with lepra vulgaris, on purpose to try the effects of the chloride of sulphur. The ease was one of six years standing, but had never during that time been completely cured; she was an in-patient in several hospitals, and obtained other professional advice, sometimes with relief for a few weeks; her head, face, and every part of the body, were entirely covered with the eruption: she used the ointment of the chloride of sulphur for six weeks, and was completely cured. 